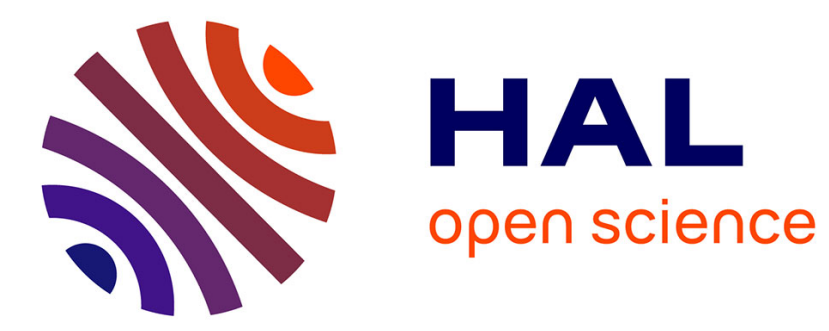

\title{
Novel cosmic-ray electron and positron constraints on MeV dark matter particles
}

\author{
Mathieu Boudaud, Julien Lavalle, Pierre Salati
}

\section{To cite this version:}

Mathieu Boudaud, Julien Lavalle, Pierre Salati. Novel cosmic-ray electron and positron constraints on MeV dark matter particles. Physical Review Letters, 2017, 10.1103/PhysRevLett.119.021103 . hal-01421563

\section{HAL Id: hal-01421563 https://hal.science/hal-01421563}

Submitted on 22 Dec 2016

HAL is a multi-disciplinary open access archive for the deposit and dissemination of scientific research documents, whether they are published or not. The documents may come from teaching and research institutions in France or abroad, or from public or private research centers.
L'archive ouverte pluridisciplinaire HAL, est destinée au dépôt et à la diffusion de documents scientifiques de niveau recherche, publiés ou non, émanant des établissements d'enseignement et de recherche français ou étrangers, des laboratoires publics ou privés. 


\title{
Novel cosmic-ray electron and positron constraints on $\mathrm{MeV}$ dark matter particles
}

\author{
Mathieu Boudaud, ${ }^{1,2}, *$ Julien Lavalle, ${ }^{3, \dagger}$ and Pierre Salati ${ }^{1, \ddagger}$ \\ ${ }^{1}$ LAPTh, Université Savoie Mont Blanc \& CNRS, \\ 9 Chemin de Bellevue, B.P.110, F-74941 Annecy-le-Vieux-France \\ ${ }^{2}$ Laboratoire de Physique Théorique et Hautes Énergies (LPTHE), \\ UMR 7589 CNRS \& UPMC, 4 Place Jussieu, F-75252 Paris - France \\ ${ }^{3}$ Laboratoire Univers \& Particules de Montpellier (LUPM), \\ CNRS \& Université de Montpellier (UMR-5299), \\ Place Eugène Bataillon, F-34095 Montpellier Cedex 05 — France
}

\begin{abstract}
$\mathrm{MeV}$ dark matter (DM) particles annihilating or decaying to electron-positron pairs cannot, in principle, be observed via local cosmic-ray (CR) measurements because of the shielding solar magnetic field. In this Letter, we take advantage of the fact that the Voyager I spacecraft, which does measure CRs, has crossed the heliopause in 2012 and can actually, since then, detect interstellar CRs. This opens up a new avenue to probe DM in the sub-GeV energy/mass range that we first exploit here. From a complete description of the transport of electrons and positrons at low energy, we derive predictions for both the secondary astrophysical background and the pair production mechanisms relevant to DM annihilation or decay down to the $\mathrm{MeV}$ mass range. Interestingly, we show that reacceleration may push positrons up to energies larger than the DM particle mass. We combine the constraints from the Voyager and AMS-02 data to get novel limits covering a very extended DM particle mass range, from $\mathrm{MeV}$ to $\mathrm{TeV}$. In the $\mathrm{MeV}$ mass range, our limits reach annihilation cross sections of order $\langle\sigma v\rangle \sim 10^{-28} \mathrm{~cm}^{3} / \mathrm{s}$. Though extracted from a completely different and new probe, these bounds have a strength similar to those obtained with the cosmic microwave background $(\mathrm{CMB})$ - they are even more stringent for p-wave annihilation.

PACS numbers: 12.60.-i,95.35.+d,96.50.S-,98.35.Gi,98.70.Sa
\end{abstract}

Thermally produced sub-GeV dark matter (DM) particles have triggered interest since the non-baryonic particle DM proposal itself, including the weakly interacting massive particle (WIMP) paradigm (e.g. [1]). Allowed scenarios involve DM particle masses $m_{\chi}$ larger than a few keV (warm DM - WDM), usually bounded by structure formation [2-5]. In the $\mathrm{MeV}$ mass range, thermal DM candidates are already cold enough not to differ from cold DM (CDM) structure formation on scales of dwarf galaxies. However, if they have remained coupled to radiation or neutrinos sufficiently long, an oscillatory damping pattern in the structure power spectrum could be observed on small scales that differs from the standard free-streaming cut-off of WDM [6], and alleviate the toobig-to-fail problem affecting the CDM paradigm on small scales [7]. Besides, self-interacting DM scenarios could be achieved from thermal freeze out of particles in the $\mathrm{MeV}$ mass range [8], as well as strongly interacting DM [9], which may also cure small scale issues in structure formation. Overall, many efforts are now devoted to probe this mass range through direct and indirect searches (see e.g. [10]).

Astrophysical observations already constrain MeV DM candidates. For instance, gamma-ray observations generically constrain $\mathrm{MeV}$ candidates depending on assumptions on the shape of the inner Galactic halo profile

\footnotetext{
* boudaud@lpthe.jussieu.fr

† lavalle@in2p3.fr

‡ pierre.salati@lapth.cnrs.fr
}

$[11,12]$. Heating of the plasma at the CMB decoupling time is also constrained by current observations and actually allows to get stringent bounds on $\mathrm{MeV}$ annihilating or decaying DM [13-15], down to an s-wave annihilation cross section of $\langle\sigma v\rangle \lesssim 10^{-29} \mathrm{~cm}^{3} / \mathrm{s}$ for the former case (assuming annihilation into electron-positron $\left[e^{ \pm}\right]$pairs).

Less prone to uncertainties in the halo shape [16], cosmic-ray $(\mathrm{CR}) e^{ \pm} \mathrm{s}$ could provide independent probes of annihilation or decay of MeV DM. Nevertheless, interstellar sub-GeV $e^{ \pm} \mathrm{S}$ are shielded by the solar magnetic field (the so-called solar modulation effect) [17, 18] such that they cannot reach detectors orbiting the Earth. In this Letter, we bypass this limitation by exploiting, for the first time in this context, the $e^{ \pm}$data of the Voyager 1 spacecraft $[19,20]$. Indeed, Voyager 1 has crossed the heliopause during the summer 2012, and, since then, has traveled through interstellar space. Since it is equipped with particle detectors, with one dedicated to $e^{ \pm}$measurements (no discrimination between electrons and positrons), this opens up a new avenue for DM searches in the sub-GeV mass range. Here, we will use the $e^{ \pm}$Voyager 1 data from end 2012, extracted after calibration of response functions from simulations of the detector (most conservative dataset) and released in Ref. [20] - i.e. 4 data points in the $\sim 10-50 \mathrm{MeV}$ energy range with excellent statistics. This dataset will be complemented at higher energy by the AMS-02 positron data [21] imported from the database proposed in [22].

The transport of $\mathrm{CR} e^{ \pm} \mathrm{S}$ in the Milky Way (MW) can be addressed by a general diffusion equation [23-29] that includes spatial diffusion, convection, reacceleration, and energy losses. We use the semi-analytic method proposed 
in Ref. [30] to solve this equation. Solutions assume a plain diffusion over a cylindrical magnetic halo of radius $R$ and half-height $L$, with boundary conditions such that the $\mathrm{CR}$ density vanishes at the halo borders. Some processes are dominant in the tiny Galactic disk, others extend to the whole magnetic halo. The first category includes diffusive reacceleration (featured by a pseudoAlfvén velocity $V_{a}$ ), and energy losses due to electromagnetic interactions with the interstellar gas $\left(b_{\text {gas }}(E)\right)$. The second one includes spatial diffusion (with a scalar coefficient $K(\mathcal{R})=\beta K_{0}(\mathcal{R} / 1 \mathrm{GV})^{\delta}$, with $\mathcal{R} \equiv p /|q|$ the rigidity), convection (with velocity $\vec{V}_{c}=\operatorname{sign}(z) V_{c} \vec{e}_{z}$ of constant modulus), and higher-energy losses from inverse Compton and synchrotron emissions $(b(E))$. The technical difficulty in applying this method to $e^{ \pm} \mathrm{s}$ comes from the fact that derivatives in momentum space are not confined to the disk as is the case for nuclei, but high-energy losses, which dominate above $\sim 10 \mathrm{GeV}$, are efficient all over the magnetic halo. This was addressed in an approximate way in Ref. [26], but recently solved in a systematic and elegant way in Ref. [29]. We therefore refer the reader to Ref. [29] for a thorough presentation of the propagation model we will further use in this Letter. Note that complementary full numerical approaches exist [31-33], which are qualitatively similar to ours.

For the propagation parameters, we consider large- $L$ propagation models because low-energy positron data (0.1-2 GeV) severely constrain values of $L \lesssim 8 \mathrm{kpc}$ $[29,34]$, as do the latest B/C data [35]. More precisely, we use the Max model proposed in $[30,36]$ (model $A$ henceforth $-L=15 \mathrm{kpc}, K_{0}=0.0765 \mathrm{kpc}^{2} / \mathrm{Myr}, \delta=0.46$, $\left.V_{a}=117.6 \mathrm{~km} / \mathrm{s}, V_{c}=5 \mathrm{~km} / \mathrm{s}\right)$, which lies at the border of the current positron bounds [29], together with the B/C best-fit model of Ref. [35] (model $B-L=13.7$ $\mathrm{kpc}, K_{0}=0.0967 \mathrm{kpc}^{2} / \mathrm{Myr}, \delta=0.407, V_{a}=31.9 \mathrm{~km} / \mathrm{s}$, $V_{c}=0.2 \mathrm{~km} / \mathrm{s}$ ). These models mostly differ in their reacceleration parameter $V_{a}$, which is large for model $A$ (fitted on old $\mathrm{B} / \mathrm{D}$ data), and small for model $B$ (most recent $\mathrm{B} / \mathrm{C}$ data) - we will see later why reacceleration is an important parameter. A full exploration of the parameter space goes beyond the scope of this Letter, but models $A$ and $B$ characterize the state-of-the-art description of Galactic CR propagation within a standard set of assumptions (isotropic and scalar spatial diffusion).

We first compute the secondary $e^{ \pm}$fluxes, i.e. $e^{ \pm} \mathrm{s}$ generated from inelastic interactions between $\mathrm{CR}$ nuclei and the interstellar gas. Though conventional sources of primary CRs (e.g. pulsar winds, supernova remnants) contribute to the total $e^{ \pm}$fluxes [27, 28, 37, 38], we only consider the secondary background because large theoretical uncertainties affect this primary component [27, 28], which puts our coming constraints on the conservative side. Our predictions for the interstellar flux are shown in Fig. 1 against the Voyager and AMS-02 data. For the latter, we need to demodulate the data (assuming the Voyager data to be modulation-free). We proceed by using the force-field approximation [17, 39] with a Fisk potential $\phi$ in the range [724MV, 830 MV] for the

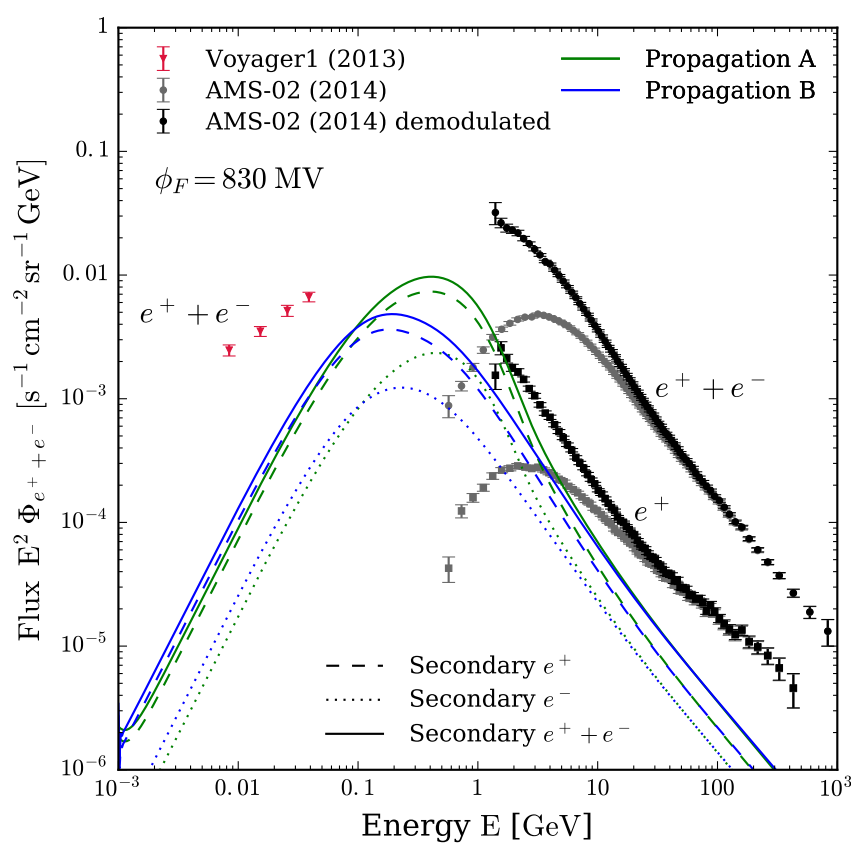

FIG. 1: CR $e^{ \pm}$data from Voyager (red triangles) and AMS-02 (plain circles), and $e^{+}$AMS-02 data (plain squares). The latter are demodulated with a Fisk potential of $\phi=830 \mathrm{MV}$. The curves show the interstellar secondary

background predicted for propagation models $A$ and $B$.

AMS-02 data taking period, indicated by Ref. [40]. From Fig. 1, we see that the secondary $e^{ \pm} \mathrm{S}$ contribute significantly to the data only in the AMS-02 energy range and are negligible in the Voyager range. This has important consequences: not only the Voyager data are free of solar modulation, but they are also insensitive to the presence of secondaries. Besides, we can already notice from Fig. 1 the impact of reacceleration: the secondary $e^{ \pm}$peak observed in $E^{2} \Phi_{e^{ \pm}}$is shifted to higher energy in the strong-reacceleration case (model $A$ ), which will make the AMS-02 data more constraining than in the weak-reacceleration case (model $B$ ). Finally, it is worth pointing out that the lowest AMS-02 energy point for the $e^{+}$flux lies significantly lower than its neighbors, which may lead to very strong bound on DM annihilation or decay. To remain conservative and since this point looks rather peculiar, we will remove it from our analysis.

We now compute the DM annihilation contributions to these observables. We consider several channels and assume that the branching ratio is unity: $e^{ \pm}, \mu^{ \pm}, \tau^{ \pm}$, $b \bar{b}$. We generate injection spectra with the Micromegas code [41], which includes final-state radiation (FSR) processes. For the DM halo profile, we assume two different spherical cases: an Navarro-Frenk-White halo [42] scaling like $1 / r$ in the center (NFW halo henceforth), and a cored halo profile with constant central DM density (cored halo). We use the corresponding constrained halo parameters from Tab. 6 of Ref. [43], which are fitted against kinematic data, such that our halos are dynam- 


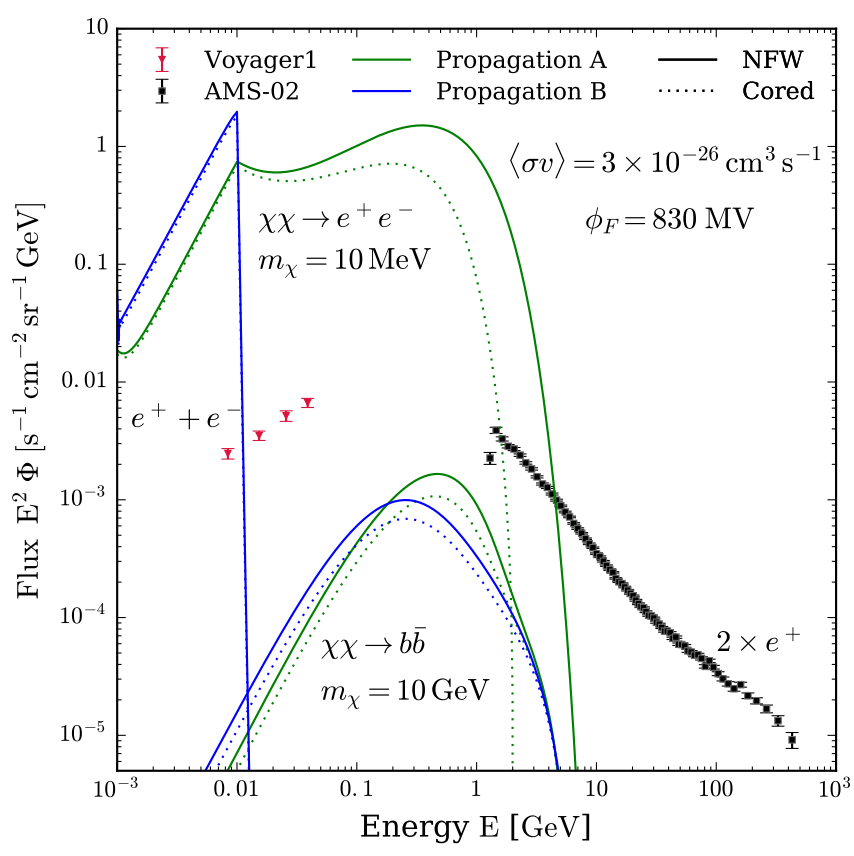

FIG. 2: Predictions $\left(e^{ \pm}\right)$for 2 template cases: a $10 \mathrm{MeV}$ WIMP annihilating into $e^{+} e^{-}$(+ FSR), and a $10 \mathrm{GeV}$ WIMP annihilating into $b \bar{b}$. The data are the same as in Fig. 1, but the AMS-02 $e^{+}$data is multiplied by a factor of 2 to compare with the $e^{ \pm}$primaries. Propagation models $A$ and $B$, and the NFW and cored DM halo models were used.

ically self-consistent. In both halos, the DM density at the solar position $r_{\odot} \simeq 8.2 \mathrm{kpc}$ is $\rho_{\odot} \simeq 0.4 \mathrm{GeV} / \mathrm{cm}^{3}$.

Template predictions for the DM-induced $e^{ \pm}$fluxes are shown in Fig. 2, considering DM particles of $10 \mathrm{MeV}$ (10 $\mathrm{GeV})$ annihilating into $e^{+} e^{-}(\gamma)(b \bar{b}$, respectively). In both cases, $e^{+} \mathrm{s}$ and $e^{-} \mathrm{s}$ share the same injection spectrum and experience the same propagation history, such that $e^{ \pm}$predictions can be compared to the $e^{+}$data by multiplying the latter by two. We reported our results for propagation models $A$ and $B$, and for the NFW and cored halos. Regarding propagation, reacceleration effects dominate at low energy. As for secondaries, strongreacceleration model $A$ leads to a much more pronounced shift of the flux to higher energy. This is clear for the $e^{+} e^{-}$channel when comparing predictions between model $A$ (strong reacceleration) and model $B$ (weak reacceleration $)^{1}$ : in the latter case, the $e^{ \pm}$flux is suppressed beyond the maximal injected energy set by $m_{\chi}$, while in the former case, low-energy $e^{ \pm} \mathrm{S}$ are reaccelerated beyond $m_{\chi}$. This is a very important feature induced by strong-reacceleration models that, to our knowledge, has remained unnoticed so far: DM annihilation-induced $e^{ \pm} \mathrm{S}$ could be observed at energies higher than $m_{\chi}$, which makes the $\mathrm{GeV}$ data also relevant to constrain sub-GeV

\footnotetext{
${ }^{1}$ The relevant (inverse) timescale to consider is given by $V_{a}^{2} / K_{0} \sim$ $0.2 \mathrm{Myr}^{-1}$ for model $A\left(0.01 \mathrm{Myr}^{-1}\right.$ for model B).
}

DM candidates. Reacceleration somehow also rules the impact of changing the DM halo shape. Indeed, in the absence of reacceleration, $e^{ \pm} \mathrm{s}$ coming from regions close to the GC have lost a significant part of their energy and populate the very low-energy part of the spectrum. Therefore, still focusing on the $e^{+} e^{-}$channel, even if an NFW halo induces a prominently larger annihilation rate toward the GC, the net increase in the $E^{2} \Phi_{e^{+}}$curve for model $B$ is lost at low energies. On the other hand, efficient reacceleration (model $A$ ) makes these $e^{+} \mathrm{s}$ continuously reheated as they cross the disk on their way to us, compensating for energy losses, such that the difference between an NFW and a cored halo is now more pronounced beyond $m_{\chi}$ (though still quite moderate with respect to corresponding changes in gamma-ray predictions). This reasoning is no longer valid above a few $\mathrm{GeV}$ where reacceleration becomes a subdominant process, as is the case in the high-energy part of the $b \bar{b}$ spectrum, but allows to understand the main qualitative features of our predictions. This non-trivial impact of reacceleration highlights the nice complementarity of the lowenergy data from Voyager with the higher-energy data from AMS-02, the former (latter) providing strong constraints on predictions based upon weak- (strong-) reacceleration models. A significant asymmetry in the constraining power of these two data sets, though, comes from the fact that the irreducible secondary background has a large contribution above $100 \mathrm{MeV}$ only $(\sim$ the charged pion mass), i.e. in the AMS-02 range.

We now combine the Voyager and AMS-02 data discussed above to derive limits on $\langle\sigma v\rangle$. We assume Majorana DM particles - a factor of 2 must be applied to our limits for Dirac fermions. We also assume that $\langle\sigma v\rangle$ is position independent (valid for an s-wave, approximate for a p-wave). We derive limits by adding our flux predictions for the primary and secondary components, and then demanding the total flux to lie below $2 \sigma$ from each data point. These limits are displayed in Fig. 3. In the left panel, we specialize to the $e^{+} e^{-}$channel to illustrate differences due to propagation, solar modulation, and the DM halo shape. As already emphasized above, the main variation is driven by reacceleration: strongreacceleration models $(\sim$ model $A$ ) induce more stringent limits, simply because sub-GeV $e^{ \pm} \mathrm{s}$ are then shifted up to the $\mathrm{GeV}$ range, in which case AMS-02 constraints are turned on - curves are thus rather smooth over the full $\mathrm{MeV}-\mathrm{TeV}$ energy range, with a transition below $\sim 10$ $\mathrm{MeV}$ where Voyager takes over. Consequently, limits inferred from strong-reacceleration models are more sensitive to solar modulation, as illustrated by decreasing $\phi=830 \mathrm{MV}$ to $724 \mathrm{MV}$ for model A: this justifies our choice of $830 \mathrm{MV}$, which is the most conservative one. In contrast, weak-reacceleration models $(\sim$ model $B)$ make it possible to "hide" a positron $E^{2} \Phi_{e^{+}}$peak in the blind spot between the two datasets, such that the $0.1-1 \mathrm{GeV}$ mass range becomes unconstrained. Nevertheless, in this case, Voyager provides severe constraints below $\sim 100$ $\mathrm{MeV}$, with the nice bonus of not suffering from solar 

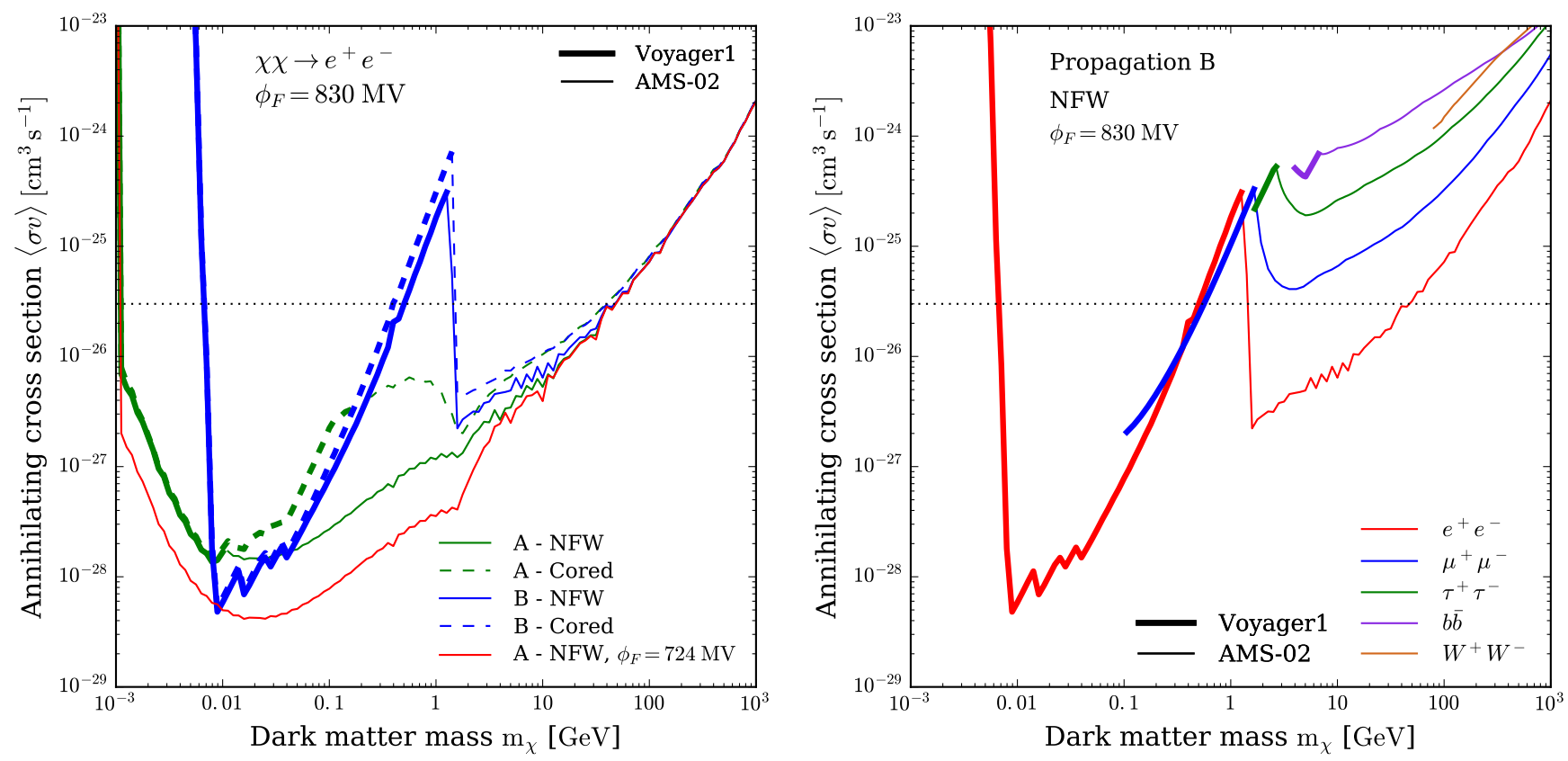

FIG. 3: Limits on $\langle\sigma v\rangle$ as a function of $m_{\chi}$. Left: limits assuming annihilation into $e^{+} e^{-}$for propagation models $A$ and $B$, and for the NFW and cored DM halos. A conservative solar modulation is set with $\phi=830 \mathrm{MV}$. The result for $\phi=724 \mathrm{MV}$ is shown for the $A$-NFW configuration. Right: limits for different annihilation final states, assuming configuration $B$-NFW-830 MV.

modulation.

In the right panel of Fig. 3, we generalize our limits for several annihilation channels conservatively assuming propagation model $B, \phi=830 \mathrm{MV}$ for the solar modulation of the AMS-02 data, and the NFW halo (closer to the best fit of [43] than the cored halo). These are our main results, which demonstrate for the first time that $\mathrm{CR} e^{ \pm} \mathrm{S}$ constrain annihilating DM down to the $\mathrm{MeV}$ mass range. We emphasize that for the $e^{+} e^{-}$channel our bound reaches $\langle\sigma v\rangle \sim 10^{-28} \mathrm{~cm}^{3} / \mathrm{s}$ in the $10-100 \mathrm{MeV}$ mass range. We also notice the blind spot just below 1 $\mathrm{GeV}$, but we stress that more reacceleration would fill in this spot again - future studies on propagation parameters will be crucial to settle this. At higher energy, we exclude thermal cross sections $\left(\sim 3 \cdot 10^{-26} \mathrm{~cm}^{3} / \mathrm{s}\right)$ for masses up to $\sim 50 \mathrm{GeV}$. This is less stringent than bounds obtained in Ref. [44], but this comes from the fact that the authors have assumed additional primaries from pulsars which saturate the data and forbids DM-induced contributions. Because of the large uncertainties affecting this primary component $[27,28]$, we have instead decided to discard it.

We now compare our results with those obtained from CMB analyses. In Ref. [15], limits on s-wave annihilations obtained for the $e^{+} e^{-}$channel go from $\langle\sigma v\rangle \lesssim$ $3 \cdot 10^{-30} \mathrm{~cm}^{3} / \mathrm{s}$ at $1 \mathrm{MeV}$ up to $\sim 3 \cdot 10^{-29} \mathrm{~cm}^{3} / \mathrm{s}$ at 100 $\mathrm{MeV}$, i.e. one order of magnitude better than ours. However, for p-wave annihilations, CMB limits degrade up to $\sim 10^{-24} \mathrm{~cm}^{3} / \mathrm{s}$ in the same mass range (derived assuming a velocity dispersion $\sigma_{v}=100 \mathrm{~km} / \mathrm{s}$ ). We can roughly convert our s-wave limits in terms of p-wave by assuming an isothermal velocity distribution for DM such that $\sigma_{v}^{\mathrm{MW}}=v_{c} / \sqrt{2}$, where $v_{c} \simeq 240 \mathrm{~km} / \mathrm{s}$ is the local rotation velocity [45]. Therefore, our s-wave bounds $\langle\sigma v\rangle_{\max }$ rescale to $\langle\sigma v\rangle_{\max }\left(\sigma_{v} / \sigma_{v}^{\mathrm{MW}}\right)^{2}$ in terms of $\mathrm{p}$-wave, giving $\sim 310^{-29} \mathrm{~cm}^{3} / \mathrm{s}$ for $\sigma_{v}=100 \mathrm{~km} / \mathrm{s}$, i.e. an improvement by $\sim 5$ orders of magnitude. Finally, our bounds are slightly more stringent than those derived in gamma-ray studies [12], and less sensitive to the DM halo shape.

To conclude, we have considered for the first time the Voyager $e^{ \pm}$data to derive constraints on annihilating MeV DM particles. Since Voyager has crossed the heliopause, solar modulation, which prevents MeV CRs to reach space experiments orbiting the Earth, can be neglected. We used state-of-the-art semi-analytic methods to describe CR propagation, including all relevant processes. We considered constrained sets of propagation parameters featuring strong (model $A$ ) and weak reacceleration (model $B$ ) to point out an interesting phenomenon: reacceleration may push $e^{ \pm}$up to energies higher than $m_{\chi}$ in the sub-GeV mass range. Thus, GeV data become constraining also for DM particles in the sub-GeV mass range. We therefore combined the Voyager and AMS-02 datasets to derive constraints on DM annihilation, getting limits down to $\langle\sigma v\rangle \sim 10^{-28} \mathrm{~cm}^{3} / \mathrm{s}$ at $10 \mathrm{MeV}$, quite competitive with respect to complementary gamma-ray studies, and less dependent on the halo shape. Other complementary CMB constraints are found more stringent for s-wave annihilations but less stringent by about five orders of magnitude for p-waves. Finally, note that a 
similar analysis could apply to heavier DM particles with excited states separated by $\mathrm{MeV}$ gaps [46].

\section{ACKNOWLEDGMENTS}

We wish to thank Alan C. Cummings for valuable exchanges about the Voyager I data, and Martin Winkler for details about the $\mathrm{B} / \mathrm{C}$ analysis made in Ref. [35]. MB acknowledges support from the European Research Council (ERC) under the EU Seventh Framework Programme (FP7/2007-2013)/ERC Starting Grant (agree- ment n. 278234 — 'NewDark' project led by M. Cirelli). JL is partly supported by the OCEVU Labex (ANR-11LABX-0060), the CNRS program Défi InPhyNiTi, and European Union's Horizon 2020 research and innovation program under the Marie Skłodowska-Curie grant agreements No 690575 and No 674896 - in addition to recurrent funding by CNRS and Montpellier University. PS is partly supported by the French Institut universitaire de France, by the French Agence Nationale de la Recherche under contract 12-BS05-0006 DMAstroLHC, by the Investissements davenir, Labex ENIGMASS, and by the PRC (Projet de Recherche Conjoint) CNRS-FAPESP.
[1] J. R. Bond, A. S. Szalay, and M. S. Turner, Physical Review Letters 48, 1636 (1982).

[2] S. Colombi, S. Dodelson, and L. M. Widrow, Astrophys. J. 458, 1 (1996), astro-ph/9505029.

[3] M. Viel, J. Lesgourgues, M. G. Haehnelt, S. Matarrese, and A. Riotto, Phys. Rev. D 71, 063534 (2005), astro$\mathrm{ph} / 0501562$.

[4] M. Viel, G. D. Becker, J. S. Bolton, and M. G. Haehnelt, Phys. Rev. D 88, 043502 (2013), arXiv:1306.2314 [astroph.CO].

[5] S. Bose, W. A. Hellwing, C. S. Frenk, A. Jenkins, M. R. Lovell, J. C. Helly, and B. Li, MNRAS 455, 318 (2016), arXiv:1507.01998.

[6] C. Bœhm, J. A. Schewtschenko, R. J. Wilkinson, C. M. Baugh, and S. Pascoli, MNRAS 445, L31 (2014), arXiv:1404.7012.

[7] M. Boylan-Kolchin, J. S. Bullock, and M. Kaplinghat, MNRAS 415, L40 (2011), arXiv:1103.0007 [astroph.CO].

[8] X. Chu, C. Garcia-Cely, and T. Hambye, ArXiv e-prints (2016), arXiv:1609.00399 [hep-ph].

[9] Y. Hochberg, E. Kuflik, T. Volansky, and J. G. Wacker, Physical Review Letters 113, 171301 (2014), arXiv:1402.5143 [hep-ph].

[10] J. Alexander et al., ArXiv e-prints (2016), arXiv:1608.08632 [hep-ph].

[11] J. F. Beacom, N. F. Bell, and G. Bertone, Physical Review Letters 94, 171301 (2005), astro-ph/0409403.

[12] R. Essig, E. Kuflik, S. D. McDermott, T. Volansky, and K. M. Zurek, Journal of High Energy Physics 11, 193 (2013), arXiv:1309.4091 [hep-ph].

[13] X. Chen and M. Kamionkowski, Phys. Rev. D 70, 043502 (2004), astro-ph/0310473.

[14] T. R. Slatyer, Phys. Rev. D 93, 023527 (2016), arXiv:1506.03811 [hep-ph].

[15] H. Liu, T. R. Slatyer, and J. Zavala, Phys. Rev. D 94, 063507 (2016), arXiv:1604.02457.

[16] J. Lavalle and P. Salati, Comptes Rendus Physique 13, 740 (2012), arXiv:1205.1004 [astro-ph.HE].

[17] L. J. Gleeson and W. I. Axford, Astrophys. J. 154, 1011 (1968).

[18] M. Potgieter, Living Reviews in Solar Physics 10, 3 (2013), arXiv:1306.4421 [physics.space-ph].

[19] S. M. Krimigis, C. O. Bostrom, T. P. Armstrong, W. I. Axford, C. Y. Fan, G. Gloeckler, and L. J. Lanzerotti, Space Science Reviews 21, 329 (1977).
[20] E. C. Stone, A. C. Cummings, F. B. McDonald, B. C. Heikkila, N. Lal, and W. R. Webber, Science 341, 150 (2013).

[21] M. Aguilar, D. Aisa, A. Alvino, G. Ambrosi, K. Andeen, L. Arruda, N. Attig, P. Azzarello, A. Bachlechner, F. Barao, and et al., Physical Review Letters 113, 121102 (2014).

[22] D. Maurin, F. Melot, and R. Taillet, Astron. Astroph. 569, A32 (2014), arXiv:1302.5525 [astro-ph.HE].

[23] V. L. Ginzburg and S. I. Syrovatskii, The Origin of Cosmic Rays, New York: Macmillan, 1964 (1964).

[24] S. V. Bulanov and V. A. Dogel, Astrophysics and Space Science 29, 305 (1974).

[25] I. V. Moskalenko and A. W. Strong, Astrophys. J. 493, 694 (1998), astro-ph/9710124.

[26] T. Delahaye, R. Lineros, F. Donato, N. Fornengo, J. Lavalle, P. Salati, and R. Taillet, Astron. Astroph. 501, 821 (2009), arXiv:0809.5268.

[27] T. Delahaye, J. Lavalle, R. Lineros, F. Donato, and N. Fornengo, Astron. Astroph. 524, A51 (2010), arXiv:1002.1910 [astro-ph.HE].

[28] M. Boudaud, S. Aupetit, S. Caroff, A. Putze, G. Belanger, Y. Genolini, C. Goy, V. Poireau, V. Poulin, S. Rosier, P. Salati, L. Tao, and M. Vecchi, Astron. Astroph. 575, A67 (2015), arXiv:1410.3799 [astro-ph.HE].

[29] M. Boudaud, E. F. Bueno, S. Caroff, Y. Genolini, V. Poulin, V. Poireau, A. Putze, S. Rosier, P. Salati, and M. Vecchi, ArXiv e-prints (2016), arXiv:1612.03924 [astro-ph.HE].

[30] D. Maurin, F. Donato, R. Taillet, and P. Salati, Astrophys. J. 555, 585 (2001), astro-ph/0101231.

[31] A. W. Strong and I. V. Moskalenko, Astrophys. J. 509, 212 (1998), astro-ph/9807150.

[32] C. Evoli, D. Gaggero, D. Grasso, and L. Maccione, JCAP 10, 018 (2008), arXiv:0807.4730.

[33] R. Kissmann, Astroparticle Physics 55, 37 (2014), arXiv:1401.4035 [astro-ph.HE].

[34] J. Lavalle, D. Maurin, and A. Putze, Phys. Rev. D 90, 081301 (2014), arXiv:1407.2540 [astro-ph.HE].

[35] R. Kappl, A. Reinert, and M. W. Winkler, JCAP 10, 034 (2015), arXiv:1506.04145 [astro-ph.HE].

[36] F. Donato, N. Fornengo, D. Maurin, P. Salati, and R. Taillet, Phys. Rev. D 69, 063501 (2004), astroph/0306207.

[37] C. S. Shen, Astrophys. J. Lett. 162, L181+ (1970).

[38] F. A. Aharonian, A. M. Atoyan, and H. J. Voelk, As- 
tron. Astroph. 294, L41 (1995).

[39] L. A. Fisk, J. Geophys. Res. 76, 221 (1971).

[40] A. Ghelfi, F. Barao, L. Derome, and D. Maurin, Astron. Astroph. 591, A94 (2016), arXiv:1511.08650 [astroph.HE].

[41] G. Bélanger, F. Boudjema, A. Pukhov, and A. Semenov, Computer Physics Communications 192, 322 (2015), arXiv:1407.6129 [hep-ph].

[42] J. F. Navarro, C. S. Frenk, and S. D. M. White, Astrophys. J. 490, 493 (1997), astro-ph/9611107.

[43] P. J. McMillan, ArXiv e-prints (2016), arXiv:1608.00971.
[44] L. Bergström, T. Bringmann, I. Cholis, D. Hooper, and C. Weniger, Physical Review Letters 111, 171101 (2013), arXiv:1306.3983 [astro-ph.HE].

[45] M. J. Reid, K. M. Menten, A. Brunthaler, X. W. Zheng, T. M. Dame, Y. Xu, Y. Wu, B. Zhang, A. Sanna, M. Sato, K. Hachisuka, Y. K. Choi, K. Immer, L. Moscadelli, K. L. J. Rygl, and A. Bartkiewicz, Astrophys. J. 783, 130 (2014), arXiv:1401.5377 [astro-ph.GA].

[46] D. P. Finkbeiner and N. Weiner, Phys. Rev. D 76, 083519 (2007), astro-ph/0702587. 